\title{
Inn Storage Ring of the INR Storage-Accelerating Complex
}

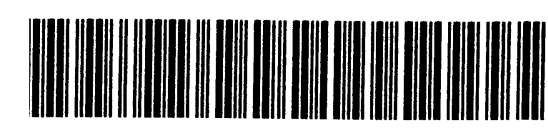

CM-P00068498

\author{
A.V. Dolinsky, A.I. Pepash, S.N. Pavlov, A.T. Rodchik, \\ A.E. Val'kov, I.N. Vishnevaky, A.V. Zhmendak \\ Institute for Naclear Research \\ 252028, Kiev, Ulorine
}

V.P. Belov, A.A. Kapustin, V.S. Kashihin, A.M. Kokorin, A.A. Matarov, B.G. Mud'yugin, B.V. Rogdestvensky, Yu.P. Severgin, IA. Schukeilo, M.N. Tarovik

D.V. Efremov Scientific Research institate of Electrophysical Apparatus 189631, St.-Petersbarg, Russia

\begin{abstract}
The Storage - Accelerating Complex (SAC) of heavy ions is intended for the storage of ions ap to Neon, their accelers tion up to an energy of $300 \mathrm{MeV} / \mathrm{a}(\mathrm{A} / Z=2)$, and physical experiment on an internal target with the electron cooling used at a bigh energy. The experiments can be performed both with aarrow and wide bears on the targets. The stored current is about $10^{9}-10^{11}$ particles, the laminosity is expected to be $10^{20}-10^{30} \mathrm{~cm}^{-2} \cdot \mathrm{sec}^{-1}$, the ion beam life-time - several tens of seconds.
\end{abstract}

\section{THE STRUCTURE OF THE COMPLEX AND ITS MAIN PARAMETERS}

The Storage Accelerating Complex of heavy ions at the Institate for Nuclear Research (Kiev, Ukraine) comprises the isochronous cyclotron U-240 used as an injector, fast booster and storage synchrotron. Two stages are anticipated for the SAC development. The storage synchrotron is planned to be constructed in the first atage to atore an ion beam op to Ne with the RF-stacking and electron cooling system used to accelerate ions to $300 \mathrm{MeV} / \mathrm{n}(\mathrm{A} / \mathrm{z}=2)$ and to operate on to the inner target at a continuous electron cooling. In the second stage a multiturn recharging injection into the storage syachrotron from U-240 will be implemented, a fast cycling $200 \mathrm{MeV} / \mathrm{n}(\mathrm{A} / 2=2)$ booster with a repetition frequency of $5 \mathrm{~Hz}$ will be constracted and repeated single-turn injection from the booster to the storege synchrotron will be realized.

The SAC will make it possible to operate with intensive beams of redioactive nuclei and ions in a wide range of masses (from proton to xenon) with an energy of up to $300 \mathrm{MeV} / \mathrm{n}(\mathrm{A} / \mathrm{z}=2)$ at a high luminosity. The SAC scheme is shown in Fig.1. Its main parmeters are given in the table 1.

\section{RING ELECTROMAGNET OF THE STORAGE SYNCHROTRON.}

It comprises two superperiods with a triplet focusing and includes eight 45 bending magnets, 36 quadrapole lenses and 8 sufficiently long rectangalar rections of 3 different types. Two of them with a zero dispersion and $\beta_{z, 2} \approx$ $2-5 \mathrm{~m}$ are intended for the installation of the electron cooling system (C3O) and acceleration stations (YC). Another two of them with a zero dispersion and low $\beta_{2,2}<0.8 \mathrm{mo}$ are designed specially for physical experiments on the target M1. The beam is focused on M1 by two triplets of the quadropole lenses JIK 46. The arrangement and param. eters of the lenses are selected so that their switching-on changes the characteristic functions only in the section, where they are installed. The rest 4 sections with the dis persion different from zero are intended to perfom physical experiments on the target $M 3(\beta<4 m, \psi * 3 \mathrm{~m})$ and to implement 3 different types of injection into the storage synchrotron, i.e. aingle and multiturn recharging injection from U-240 with the electron cooling and RF-stacking used and single-tarn injection from the booster. Eight sextupole lenses are used for chromsticity correction.

\section{INJECTION AND STORAGE.}

An average carrent of ion beams from the cyclotron U-240 varies from $0.2 \mu \mathrm{A}(\mathrm{Ne})$ to $10 \mu \mathrm{A}(\mathrm{P})$. With the $\mathrm{ECR}$. source used the ion hesvier than $\mathrm{Xe}$ can be accelerated in cyclotron. The operating cycle of the storage synchrotron (without the booster) is the following:

1. the single-turn injection of light element nuclei or maltiturn recharging injection (of about $40 \mu \mathrm{A}$ duration) of hesvy ions;

2. RF-stacking (of 10-20 ms duration) with an increase in the energy of the injected beam by $2.3 \%$ (single-turn injection) or by $1.2 \%$ (maltiturn recharging injection). The RF-stacking is used to eliminate the cooled beam travel through the inpot kicker-msgnet or the stripping target M2 with, respectively, these two types of injection used;

3. electron cooling of the injected beam during 40 $1400 \mathrm{~ms}$;

4. storage resulting from the multiple repetition of the operation according to the above points $1,2,3$ (dtration of up to 10 and more); 
Table 1: The SAC parameters

\begin{tabular}{|l|c|c|}
\hline & itorage-synchrotron & booster \\
\hline Perimeter, m & 106.275 & 57.452 \\
Nomber of betatron oscillations & $3.709 / 3.406$ & $1.8 / 1.288$ \\
Magnet field indaction, T & 1.5 & 1.5 \\
Maximum magnet rigidity, T.m & 5.4 & 4.3 \\
Injection energy from U-240, Mev/n & & \\
light ions & $25 \cdot 70$ & $25 \cdot 70$ \\
C - Ar & $5 \cdot 10$ & $5 \cdot 10$ \\
Kr Xe & 3 & 3 \\
Maximum energy, Mev/n & & \\
protons & 930 & 680 \\
ions with A/z=2 & 300 & 200 \\
ions with A/2=3 & 144 & 94 \\
Vacoura, Tort & lower than $10^{-10}$ & $10^{-0}$ \\
\hline
\end{tabular}

5. acceleration with a duration of 18 ;

6. 8 physical experiment ( $\geq 10$ 8) with the electron cooling at high energy;

7. preparation of a new cycle of $\approx 18$.

To store light ione in the storage synchrotron the sngletrun injection is used. It is used as well to inject the beam into the booster. The injection of not completely stripped jons with $A / z=3-5$ is implemented with the stripping on the foil. The beam is injected onto the closed orbit in the septom-magnet area After 10-15 torns the distarbance is removed for about a period of revolution and electron cooling system is switched on [1]. The beam is compressed. With a special RF-resonator used, its energy is increased by $1.2 \%$ and the closed orbit is displaced out wards by $3.7 \mathrm{~cm}$. Then, RF is repidly reset, the cooled beam leaves the resonance with the RF-field and moves along the displaced orbit, where $\boldsymbol{\phi}=0$. The injection cy. cle is repeated.

The life time of the ion heavier than Ne is 1 o at a pressure in the vecurm chamber of $<10^{-10}$ Tors and energies achievable on the cyclotron U-240. That is why, their otorage is possible only with the booster used. The total storage time in this case amonate to 46 \& (Kr), 20 s (Xe). With the operation on the inner target the ion life time is determined mainly by a single scattering on the target and the electron captare by the target atom, as the maltiple processes are suppressed by the electron cooling. The target thickness should not exceed $2.6 \cdot 10^{-10} / \mathrm{z}_{4} \mathrm{~cm}^{-2} 80$ as to compensate energy loses in the target by the electron cooling. A typical life time of heavy ions with a plambam target is about 20 8. The cooled beam with a maximam energy has an emittance of $2.4 \pi \mathrm{mm} \cdot \mathrm{mrad}$ for protons $\left(N=10^{11}\right)$ and $0.2 \pi \mathrm{mm} \cdot \mathrm{mrad}$ for $\mathrm{Ne}$ ions $\left(N=10^{9}\right)$ and a size on the target of $2.8 \mathrm{~mm}$ and $0.6 \mathrm{~mm}$ for $\mathrm{P}$ and $\mathrm{Ne}$ ions, respectively.

\section{REFERENCES}

[1] N.S. Dikankky et a. "Predelnge vozmozbnooti elektronnogo okhlazhdenigs", Preprint N 88-81, Mar 30 AN SSSR, Norosibirk, 1988. 


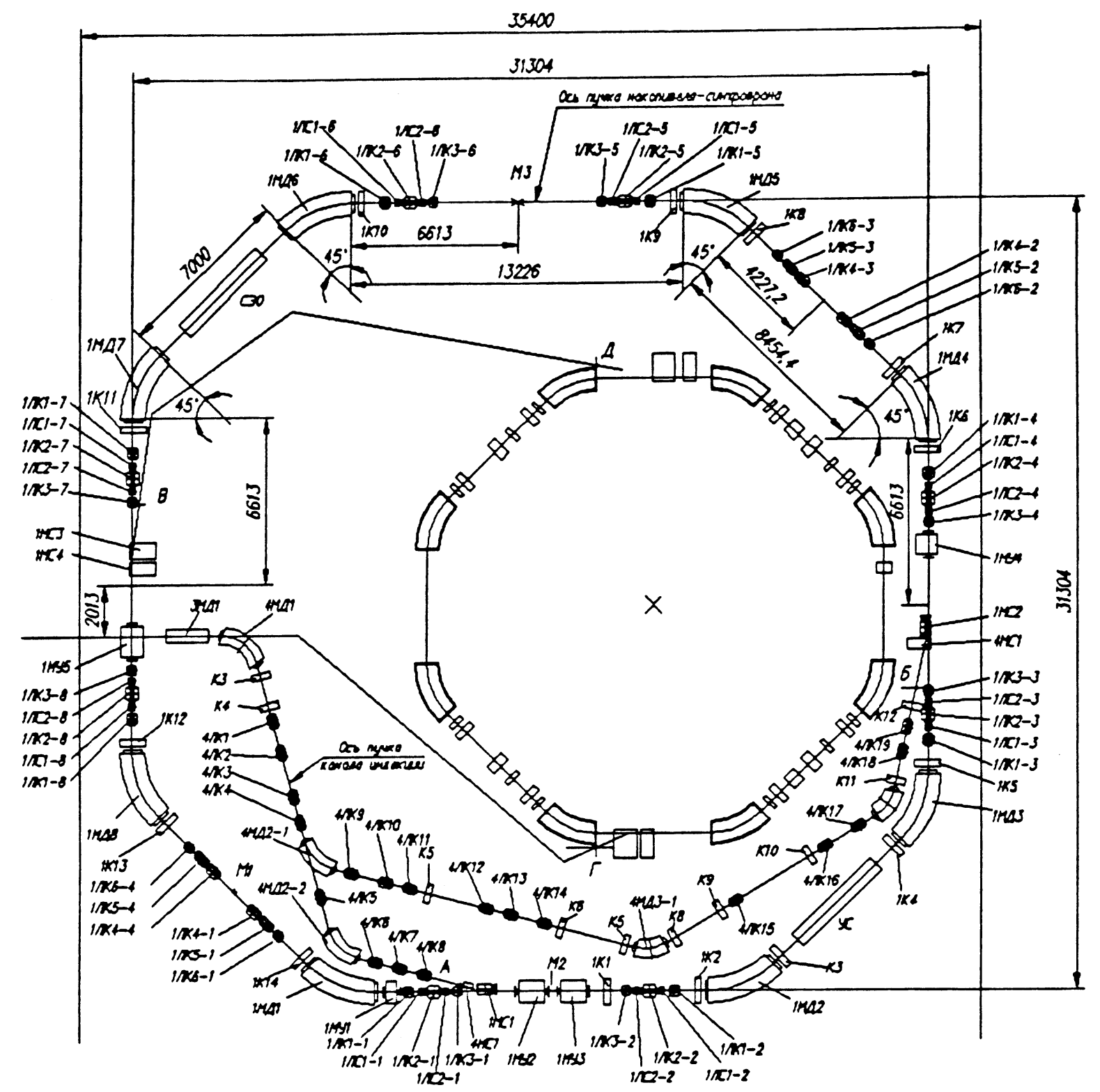

Fig.1. The INR (Kiey) Storoge-Acceleroting Complex 